\title{
A Rare Case of Right Lower Lobe Intralobar Pulmonary Sequestration in an Adult
}

Andrew J. Weaver, MD', Jenalee Corsello, MD', Peter Chirico, MD', Mark H. Cooper, MD, PhD' ${ }^{1}$

Pulmonary sequestration is a rare congenital abnormality that is often article.

diagnosed in childhood. There are two types of sequestration which are differentiated by the presence, or lack of, pleural lining dividing the aberrant lung tissue from the functional lung. Data for the management of this pathology in adults remains scarce. Here we present a case of intralobar pulmonary sequestration in an adult. The management of this patient comprised of surgical treatment involving lobectomy and ligation of the feeding vessel. The patient had an uneventful recovery, and the final pathology revealed a chronic infection in the sequestration.

\section{KEYWORDS}

Pulmonary sequestration, Right lower lobe lobectomy

\section{INTRODUCTION}

Pulmonary sequestration is a rare congenital abnormality of the lung that has been known for over 100 years. It was first described by Rektorzik in 1861 but was further elucidated as a clinical and pathological condition by Pryce in $1946 .^{1}$ The abnormality is one in which a non-functional segment or lobe of the lung is separated from the normal bronchopulmonary tree and receives an aberrant arterial supply and venous drainage. The aberrant blood supply most often arises from the thoracic (75\%) or abdominal (20\%) aorta but may also arise from smaller thoracic or abdominal arteries. ${ }^{2}$ There are two types of pulmonary sequestration. Intralobar sequestration is more common and is when the abnormal lung tissue shares a common pleura with the lung. Extralobar sequestration is when the aberrant tissue is separated from the remaining lung tissue by its own pleural investment. ${ }^{3}$ Most pulmonary sequestrations are diagnosed in early childhood. The more common intralobar sequestrations are usually in the lower lobes and may present with recurrent pneumonia, with radiographic evidence of a consolidative mass in the lower lobe. Here we present a case of intralobar pulmonary sequestration in an adult, which presented with an insidious onset but classical signs on CT scan, and the accompanying successful surgical treatment.

\section{CASE REPORT}

The patient was a 42-year-old female that was referred to thoracic surgery due to a long-standing diagnosis of pulmonary sequestration that was found incidentally on CT scan in 2009. CT scan of the chest showed pneumonia with findings of right lower lobe sequestration. At that time, she was experiencing fatigue with some shortness of breath during light activity. As a school teacher, she experienced further fatigue over the ensuing years with some mild chest discomfort. She presented again in 2020 to the thoracic surgery clinic. CT scan revealed a systemic artery arising from the right lateral aspect of the aorta, which terminated in an intralobar pulmonary sequestration (Figure 1). The sequestration drained back into the inferior pulmonary vein. The sequestration involved the posterior basal, lateral 


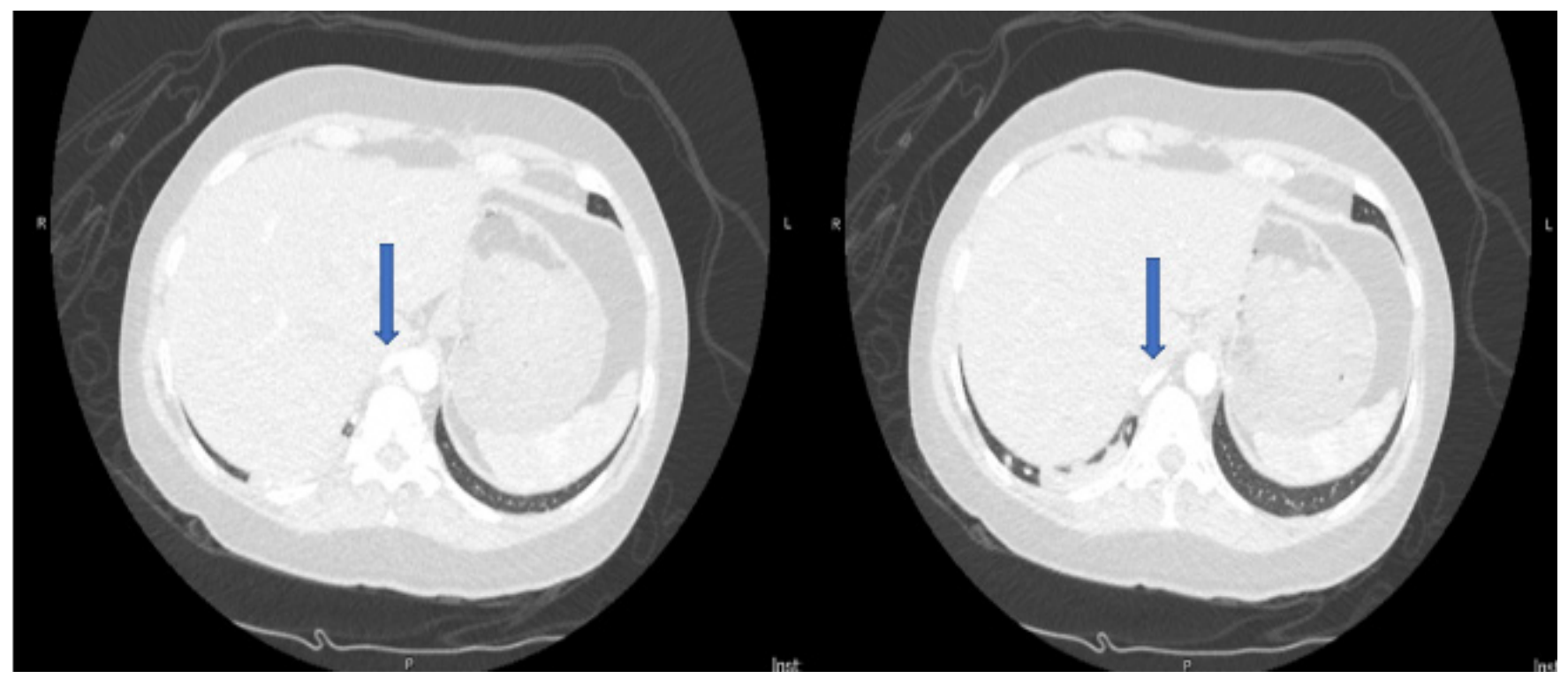

FIGURE 1. Axial CT scan of chest with IV contrast demonstrating take off of feeding vessel of the sequestration coming off the aorta.

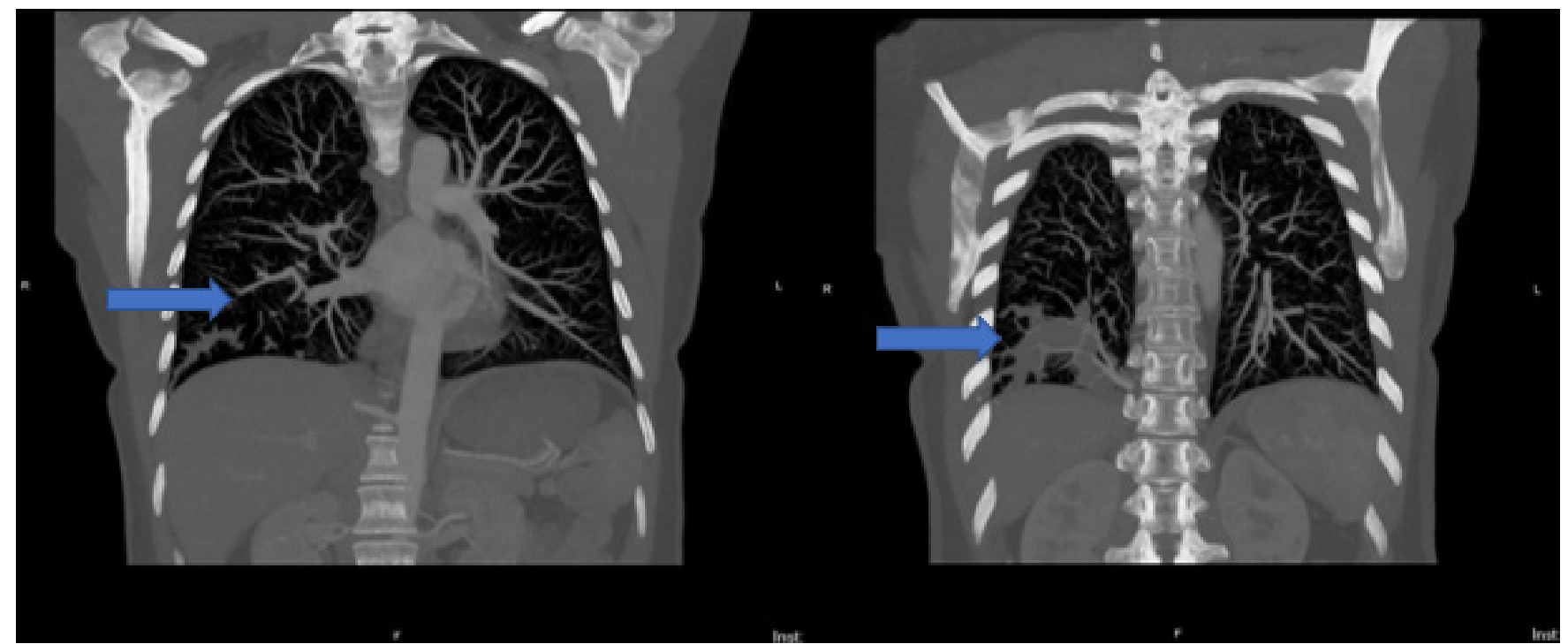

FIGURE 2. Axial CT scan of chest with IV contrast further demonstrating the separate blood supply of the sequestration.

basal, and some of the medial basal segments of the right lower lobe of her lung (Figure 2).

A pre-operative workup found her pulmonary function tests were within normal limits. She underwent a ventilation-perfusion scan which demonstrated no segmental or subsegmental defects. She also underwent a bronchoscopy pre-operatively and was noted to have no airway abnormalities, no congenital abnormalities, nor any areas of bleeding nor telangiectasia, specifically in the right lower lobe. Washings were taken and aspirated and sent off for tuberculosis, fungal, and routine cultures which showed no growth. Clinical examination was within normal limits.

On the day of surgery, she underwent a paravertebral block, intubation with a doublelumen tube for ventilation, and general anesthesia for the procedure. The surgery was approached from a standard right posterolateral thoracotomy. Dissection showed that the main branch of the 
pulmonary artery fed into the lower lobe of the right lung, whereas the sequestered area in the lower lobe was fed from an artery coming directly through the diaphragm into the mediastinum and the lower lobe. This aberrant artery was gently encircled using a vessel loop. With as minimal manipulation as possible, the aberrant artery was stapled off, followed by the inferior pulmonary vein and pulmonary artery. The dissection was completed around the lower right lobe fissures, and the bronchus, as well as lung tissue, were stapled. Mediastinal lymph node dissection was performed. An ON-Q pump was left for pain control at the end of the case.

Intraoperative findings were consistent with the CT scan findings of the patient having a right lower lobe pulmonary sequestration with feeding vessel from the abdominal aorta through the diaphragm into the mediastinum and the lower lobe directly with symptoms. Final pathology was consistent with the clinical impression of pulmonary sequestration. She did very well postoperatively and was discharged home after an uncomplicated hospital course.

\section{DISCUSSION}

The diagnosis of pulmonary sequestration is often made in childhood or early in adulthood as it presents with frequent infections or, more rarely, hemoptysis. ${ }^{4,5}$ The exact etiology is not known, but it is suggested that it is the result of an abnormal accessory tracheobronchial bud evaginating from the foregut. ${ }^{6}$ Intralobar pulmonary sequestration, as with our patient, shows no preference for one side of the thorax versus the other. Extralobar pulmonary sequestration is reported to be on the left side in up to $80 \%$ of cases. The exact etiology of side preference is not known, and bilateral sequestration is rare. 7 Intralobar pulmonary sequestration accounts for $75-90 \%$ of sequestrations, with $20 \%$ occurring in the right lower lobe. The extent of sequestrations is variable. ${ }^{8}$

Most patients with pulmonary sequestration will remain asymptomatic which makes the true incidence difficult to discern. Of symptomatic patients, typical presenting symptoms include chronic cough, chest pain, recurrent pneumonia, pulmonary abscess, as well as hemoptysis. ${ }^{9}$ For unknown reasons, but likely due to the nonfunctionality of the tissue, the sequestered tissue is prone to infections. Congestive heart failure has been reported in the literature, which is due to left to right shunting.

Radiologic manifestations of pulmonary sequestration include recurrent focal airspace disease, a parenchymal mass, and air or fluidfilled cystic lesions. Intralobar sequestration typically manifests radiologically as a homogenous consolidation with irregular margins. Angiography and MR imaging modalities can also help define the anomalous vascular supply for surgical planning. ${ }^{10}$

It is generally accepted that most patients with pulmonary sequestration should be treated operatively with lobectomy regardless of symptoms. This is in part due to the risk of developing recurrent infections. This, however, remains a source of debate. A recent retrospective review of pulmonary sequestration comparing resection versus observation was not able to determine the benefit of one over the other. The paper reported that various factors, including clinical manifestations related to pulmonary sequestration, risk of surgical complications, comorbidities, and individual patient preferences, need to be considered on a case-bycase basis. $^{5}$

A more recent treatment modality for pulmonary sequestrations is transarterial embolization. Reported cases have utilized a combination of polyvinyl alcohol (PVA) particles, gel foam, and coils with good success. ${ }^{4}$ Embolization works by occluding the arterial supply leading to sequestration infarction and is an alternative treatment method to conventional surgical resection. Some concerns have been risen from these techniques due to pain and risk of infection of infarcted pulmonary tissue. Embolization can also be used pre-operatively to decrease the risk of intraoperative hemorrhage, but its benefit remains theoretical. ${ }^{11}$

The patient in the present study was treated surgically and did well. On pathological analysis of the resected specimen, she did have evidence of continued acute on chronic infection. Her symptoms, however, were deceptively few. It could only be 
supposed that the infection would eventually progress into a more serious issue should the sequestration have been treated conservatively. Discussions with the patient have confirmed our impressions, and having a good outcome has confirmed our analysis of the risk-to-reward ratio.

\section{CONCLUSION}

Pulmonary sequestration is a rare pathological finding that, when diagnosed in adulthood, is often found incidentally on imaging but should be suspected in patients with recurrent pneumonia. It is diagnosed definitively with imaging modalities and is most often treated with surgical resection. A pre-operative CT scan of the thorax with IV contrast is vitally important for surgical planning to avoid injuring the aberrant vessels during the operation. The decision to operate needs to be multifactorial with considerations of the patient's clinical manifestations, risk of surgical complications, comorbidities, and patient preference evaluated on a case-by-case basis.

\section{AUTHOR AFFILIATIONS}

1. Marshall University Joan C. Edwards School of Medicine, Huntington, West Virginia

\section{REFERENCES}

1. Sade RM, Clouse M, Ellis FH, Jr. The spectrum of pulmonary sequestration. Ann Thorac Surg. 1974;18(6):644-58.

2. Pikwer A, Gyllstedt E, Lillo-Gil R, Jonsson P, Gudbjartsson T. Pulmonary sequestration--a review of 8 cases treated with lobectomy. Scand J Surg. 2006;95(3):190-4.

3. Sun $X$, Xiao Y. Pulmonary sequestration in adult patients: a retrospective study. Eur J Cardiothorac Surg. 2015;48(2):279-82.

4. Zener R, Bottoni $D$, Zaleski A, Fortin D, Malthaner $\mathrm{RA}$, Inculet RI, et al. Transarterial embolization of intralobar pulmonary sequestration in a young adult with hemoptysis. J Thorac Dis. 2017;9(3):E188-E93.

5. Alsumrain M, Ryu JH. Pulmonary sequestration in adults: a retrospective review of resected and unresected cases. BMC Pulm Med. 2018;18(1):97.

6. Wani SA, Mufti GN, Bhat NA, Baba AA. Pulmonary Sequestration: Early Diagnosis and Management. Case Rep Pediatr. 2015;2015:454860.

7. Savic B, Birtel FJ, Tholen W, Funke HD, Knoche R. Lung sequestration: report of seven cases and review of 540 published cases. Thorax. 1979;34(1):96-101.

8. Rao DS, Barik R. Rare presentation of intralobar pulmonary sequestration associated with repeated episodes of ventricular tachycardia. World J Cardiol. 2016;8(7):432-5.

9. Chakraborty RK, Modi P, Sharma S. Pulmonary Sequestration. [Updated $2021 \mathrm{Jul}$ 26]. In: StatPearls [Internet]. Treasure Island (FL): StatPearls Publishing; 2021 Jan-. Available from: https://www.ncbi.nlm.nih.gov/books/ NBK532314/

10. Frazier AA, Rosado de Christenson ML, Stocker JT, Templeton PA. Intralobar sequestration: radiologic-pathologic correlation. Radiographics. 1997;17(3):725-45.

11. Ojha V, Samui PP, Dakshit D. Role of endovascular embolization in improving the quality of life in a patient suffering from complicated intralobar pulmonary sequestration - A case report. Respir Med Case Rep. 2015;16:24-8. 